\title{
An asymptotic formula of a sum function involving ged and characteristic function of the set of $r$-free numbers
}

\author{
Mihoub Bouderbala ${ }^{1}$, Meselem Karras $^{2, *}$ \\ ${ }^{1}$ Institute of Mathematics, LA3C, Houari-Boumédiène University of Science and Technology, Bab Ezzouar, Algeria \\ ${ }^{2}$ FIMA Laboratory, Djilali Bounaama University, Khemis Miliana, Algeria
}

(Received: 15 August 2020. Received in revised form: 31 August 2020. Accepted: 3 September 2020. Published online: 5 October 2020.)

(C) 2020 the authors. This is an open access article under the CC BY (International 4.0) license (www.creativecommons.org/licenses/by/4.0/).

\section{Abstract}

Let $\operatorname{gcd}(k, j)$ be the greatest common divisor of the positive integers $k$ and $j$. For any real number $x>1$ and for any fixed positive integers $s$ and $r$, we give an asymptotic formula of the sum function

$$
\sum_{k \leq x} \frac{1}{k^{s+1}} \sum_{j=1}^{k} j^{s} \mu_{r}(\operatorname{gcd}(j, k)),
$$

where $\mu_{r}$ is the characteristic function of the set of $r$-free numbers.

Keywords: gcd-sum function; Dirichlet convolution; sum function.

2020 Mathematics Subject Classification: 11A25, 11 N37.

\section{Introduction}

Let $k$ and $j$ be two positive integers. We denote by $\operatorname{gcd}(k, j)$ the greatest common divisor of the integers $k$ and $j$. For any two arithmetical functions $f$ and $g$, let us consider the sum function

$$
S_{k}(j)=S_{f, g}(k, j):=\sum_{d \mid \operatorname{gcd}(k, j)} f(d) g(k / d) .
$$

The function given in (1) is a generalization of the following sum function

$$
S_{f, g}(k):=\sum_{d \mid k} f(d) g(k / d)=(f * g)(k),
$$

where the symbol “*” denotes the Dirichlet convolution of arithmetic functions. We remark here that Anderson and Apostol [1] are the first who created this sum function. However, the function $S_{k}(j)$ has been studied by several researchers, including Johnson [6], Apostol [2]; and Kiuchi, Minamide and Ueda [9]. In particular, Kiuchi [8] proved the following formula

$$
\sum_{k \leq x} \frac{1}{k^{s+1}} \sum_{j=1}^{k} j^{s} S_{k}(j)=\frac{1}{2} \sum_{n \leq x} \frac{(f * g)(n)}{n}+\frac{1}{s+1} \sum_{n \leq x}\left(\frac{f}{I d} * g\right)(n)+\frac{1}{s+1} \sum_{m=1}^{\lfloor s / 2\rfloor}\left(\begin{array}{c}
s+1 \\
2 m
\end{array}\right) B_{2 m} \sum_{n \leq x}\left(\frac{f}{I d} * \frac{g}{I d_{2 m}}\right)(n)
$$

which is valid for any positive integer $k$ and any fixed positive integer $s$, where $B_{m}$ is Bernoulli's number, $\lfloor t\rfloor$ is the integer part of $t$ and for any positive integer $n$ the functions $I d, I d_{m}$ and the unit function 1 are given as $I d(n)=n, I d_{m}(n)=n^{m}$, for any real number $m$, and $1(n)=1$. We note that the formula (2) has a lot of interesting applications (see [7]). The sums of the form

$$
\sum_{n \leq x} \sum_{j=1}^{n} f(\operatorname{gcd}(j, n))
$$

have also been studied by many researchers (see [3,4]). In 2010, O. Bordellès [4] gave an asymptotic formula of (3) under the assumption that $x \geq 1$ is sufficiently large and $f$ is an arithmetic function satisfying certain hypotheses. In this note, an asymptotic formula of the sum function

$$
\sum_{k \leq x} \frac{1}{k^{s+1}} \sum_{j=1}^{k} j^{s} \mu_{r}(\operatorname{gcd}(j, k))
$$

is given, where $x$ is any real number greater than $1 ; s$ and $r$ are any fixed positive integers; and $\mu_{r}$ is the characteristic function of the set of $r$-free numbers. 


\section{Main result}

Let $\mu_{r}$ and $f_{r}$ be two functions defined by

$$
f_{r}(n)= \begin{cases}\mu(m) & \text { if } n=m^{r} \\ 0 & \text { otherwise }\end{cases}
$$

and

$$
\mu_{r}(n)= \begin{cases}1 & \text { if } n \text { is an } r \text {-free number, } \\ 0 & \text { otherwise }\end{cases}
$$

where $r \geq 2$ is a fixed integer and $\mu$ is the Möbius function. Denote by $\zeta(s)$ the Riemann zeta-function. The proof of the next lemma is well-known, however, we include it for the sake of completeness.

Lemma 2.1. For any fixed integer $r \geq 2$, we have

$$
\mu_{r}=1 * f_{r} .
$$

i.e.,

$$
\sum_{d^{r} \mid n} \mu(d)=\mu_{r}(n)
$$

Proof. The function $f_{r}$ is clearly multiplicative, so the function $\mu_{r}$, being the convolution product of two multiplicative functions is also a multiplicative function. Therefore, it suffices to show that

$$
\mu_{r}\left(p^{\alpha}\right)=\left(1 * f_{r}\right)\left(p^{\alpha}\right)
$$

for all prime powers $p^{\alpha}$. Indeed, one has

$$
\begin{aligned}
\left(1 * f_{r}\right)\left(p^{\alpha}\right) & =\sum_{\alpha=0}^{r} f_{r}\left(p^{\alpha}\right)=1+\sum_{\alpha=1}^{r} f_{r}\left(p^{\alpha}\right) \\
& = \begin{cases}1 & \text { if } \alpha<r \\
0 & \text { if } \alpha \geq r\end{cases} \\
& =\mu_{r}\left(p^{\alpha}\right)
\end{aligned}
$$

We use the identity (5) and the formula (2) to give an asymptotic formula of the following sum

$$
\sum_{k \leq x} \frac{1}{k^{s+1}} \sum_{j=1}^{k} j^{s} \mu_{r}(\operatorname{gcd}(j, k)) .
$$

Now, we can state our main result.

Theorem 2.1. For any positive real number $x>1$ and any fixed positive integer $s$, we have

$$
\sum_{k \leq x} \frac{1}{k^{s+1}} \sum_{j=1}^{k} j^{s} \mu_{r}(\operatorname{gcd}(k, j))=\frac{x}{(s+1) \zeta(2 r)}+\frac{\log x}{2 \zeta(r)}+L(r ; s)-\frac{1}{s+1} \sum_{d^{r} \leq x} \frac{\mu(d)}{d^{r}} \psi\left(\frac{x}{d^{r}}\right)+\mathcal{O}\left(x^{-1+\frac{1}{r}} \log x\right)
$$

where

$$
L(r ; s)=\frac{1}{2(s+1) \zeta(r)}\left((s+1)\left(\gamma-\frac{r \zeta^{\prime}(r)}{\zeta(r)}\right)-1+2 \sum_{m=1}^{\left\lfloor\frac{s}{2}\right\rfloor}\left(\begin{array}{c}
s+1 \\
2 m
\end{array}\right) B_{2 m} \zeta(2 m)\right) .
$$

In order to prove Theorem 2.1, we firstly need to prove some lemmas.

Lemma 2.2. For any real number $x>1$ and any fixed positive integer s, we have

$$
\sum_{k \leq x} \frac{1}{k^{s+1}} \sum_{j=1}^{k} j^{s} \mu_{r}(\operatorname{gcd}(k, j))=\frac{1}{2} \sum_{n \leq x} \frac{\mu_{r}(n)}{n}+\frac{1}{s+1} \sum_{d l \leq x} \frac{f_{r}(d)}{d}+\frac{1}{s+1} \sum_{m=1}^{\lfloor s / 2\rfloor}\left(\begin{array}{c}
s+1 \\
2 m
\end{array}\right) B_{2 m} \sum_{d l \leq x} \frac{f_{r}(d)}{d} \frac{1}{l^{2 m}} .
$$


Proof. By using the two formulas (1), (5); and by using the definition (4), we get

$$
\begin{aligned}
S_{k}(j) & =S_{f_{r}, 1}(k, j)=\sum_{d \mid \operatorname{gcd}(k, j)} f_{r}(d) \\
& =\sum_{d^{r} \mid \operatorname{gcd}(k, j)} \mu(d) \\
& =\mu_{r}(\operatorname{gcd}(k, j)) .
\end{aligned}
$$

Thus,

$$
\sum_{k \leq x} \frac{1}{k^{s+1}} \sum_{j=1}^{k} j^{s} S_{f_{r}, 1}(k, j)=\sum_{k \leq x} \frac{1}{k^{s+1}} \sum_{j=1}^{k} j^{s} \mu_{r}(\operatorname{gcd}(k, j)) .
$$

On the other hand, the right side of (6) is a direct application of formula (2) when $f=f_{r}$ and $g=1$.

Lemma 2.3. For any $x>1$ and $r \geq 2$, we have

$$
\sum_{n \leq x} \frac{\mu_{r}(n)}{n}=\frac{\log x}{\zeta(r)}+\frac{\gamma}{\zeta(r)}-r \frac{\zeta^{\prime}(r)}{\zeta^{2}(r)}+\mathcal{O}\left(x^{-1+\frac{1}{r}} \log x\right) .
$$

Proof. Using the identity (3), and the known formula

$$
\sum_{n \leq x} \frac{1}{n}=\log x+\gamma+\mathcal{O}\left(x^{-1}\right),
$$

we have

$$
\begin{aligned}
\sum_{n \leq x} \frac{\mu_{r}(n)}{n} & =\sum_{n \leq x}\left(\frac{f_{r}}{I d} * \frac{1}{I d}\right)(n) \\
& =\sum_{d \leq x} \frac{f_{r}(d)}{d} \sum_{m \leq x / d} \frac{1}{m} \\
& =\sum_{d^{r} \leq x} \frac{\mu(d)}{d^{r}} \sum_{m \leq x / d^{r}} \frac{1}{m} \\
& =\log x \sum_{d=1}^{\infty} \frac{\mu(d)}{d^{r}}-r \sum_{d=1}^{\infty} \frac{\mu(d) \log d}{d^{r}}+\gamma \sum_{d=1}^{\infty} \frac{\mu(d)}{d^{r}}+A(x),
\end{aligned}
$$

where

$$
A(x)=\log x \sum_{d>x^{1 / r}} \frac{\mu(d)}{d^{r}}-r \sum_{d>x^{1 / r}} \frac{\mu(d) \log d}{d^{r}}+\gamma \sum_{d>x^{1 / r}} \frac{\mu(d)}{d^{r}}+\mathcal{O}\left(\frac{d^{r}}{x} \sum_{d^{r} \leq x} \frac{\mu(d)}{d^{r}}\right) .
$$

By the known identity

$$
\frac{1}{\zeta(r)}=\sum_{d=1}^{\infty} \frac{\mu(d)}{d^{r}}
$$

where $r>1$, we have

$$
\frac{\zeta^{\prime}(r)}{\zeta^{2}(r)}=\sum_{d=1}^{\infty} \frac{\mu(d) \log d}{d^{r}}
$$

and by using the estimate

$$
\sum_{n>x} \frac{1}{n^{r}}=\mathcal{O}\left(x^{-1+r}\right)
$$

where $r>1$, we get

$$
\sum_{n \leq x} \frac{\mu_{r}(n)}{n}=\frac{\log x}{\zeta(r)}+\frac{\gamma}{\zeta(r)}-r \frac{\zeta^{\prime}(r)}{\zeta^{2}(r)}+\mathcal{O}\left(x^{-1+\frac{1}{r}} \log x\right)
$$

Lemma 2.4. For any $x>1$ and $r \geq 2$, we have

$$
\sum_{d l \leq x} \frac{f_{r}(d)}{d}=\frac{x}{\zeta(2 r)}-\sum_{d^{r} \leq x} \frac{\mu(d)}{d^{r}} \psi\left(\frac{x}{d^{r}}\right)-\frac{1}{2 \zeta(r)}+\mathcal{O}\left(x^{\frac{1}{r}-1}\right) .
$$


Proof. For $x>1$ and $r \geq 2$, we have

$$
\begin{aligned}
\sum_{d l \leq x} \frac{f_{r}(d)}{d} & =\sum_{d^{r} l \leq x} \frac{\mu(d)}{d^{r}} \\
& =\sum_{d^{r} \leq x} \frac{\mu(d)}{d^{r}} \sum_{l \leq x / d^{r}} 1 \\
& =\sum_{d^{r} \leq x} \frac{\mu(d)}{d^{r}}\left\lfloor\frac{x}{d^{r}}\right\rfloor,
\end{aligned}
$$

Using the fact that

we get

$$
\psi(x)=x-\lfloor x\rfloor-\frac{1}{2}
$$

$$
\begin{aligned}
\sum_{d l \leq x} \frac{f_{r}(d)}{d} & =x \sum_{d^{r} \leq x} \frac{\mu(d)}{d^{2 r}}-\sum_{d^{r} \leq x} \frac{\mu(d)}{d^{r}} \psi\left(\frac{x}{d^{r}}\right)-\frac{1}{2} \sum_{d^{r} \leq x} \frac{\mu(d)}{d^{r}} \\
& =\frac{x}{\zeta(2 r)}-\sum_{d^{r} \leq x} \frac{\mu(d)}{d^{r}} \psi\left(\frac{x}{d^{r}}\right)-\frac{1}{2 \zeta(r)}+\mathcal{O}\left(x^{\frac{1-r}{r}}\right)
\end{aligned}
$$

Lemma 2.5. For any $x>1$ and for the two fixed integers $r \geq 2, m \geq 0$, we have

$$
\sum_{d l \leq x} \frac{f_{r}(d)}{d} \frac{1}{l^{2 m}}=\frac{\zeta(2 m)}{\zeta(r)}+\mathcal{O}\left(x^{-1+1 / r}\right)+\mathcal{O}\left(x^{-2 m / r+1 / r}\right)
$$

Proof. For $x>1$ and $r \geq 2$, we have

$$
\begin{aligned}
\sum_{d l \leq x} \frac{f_{r}(d)}{d} \frac{1}{l^{2 m}} & =\sum_{d^{r} l \leq x} \frac{\mu(d)}{d^{r}} \frac{1}{l^{2 m}} \\
& =\sum_{d \leq x^{1 / r}} \frac{\mu(d)}{d^{r}} \sum_{l \leq x^{1 / r} / d} \frac{1}{l^{2 m}} \\
& =\sum_{d \leq x^{1 / r}} \frac{\mu(d)}{d^{r}}\left(\zeta(2 m)+\mathcal{O}\left(\left(\frac{x^{1 / r}}{d}\right)^{1-2 m}\right)\right) \\
& =\frac{\zeta(2 m)}{\zeta(r)}+\mathcal{O}\left(x^{-1+1 / r}\right)+\mathcal{O}\left(x^{-2 m / r+1 / r}\right)
\end{aligned}
$$

Now, we are ready to prove the main result of this note.

Proof of Theorem 2.1. By substituting formulas (7), (8), (9) in (6), we obtain

$$
\sum_{k \leq x} \frac{1}{k^{s+1}} \sum_{j=1}^{k} j^{s} \mu_{r}(\operatorname{gcd}(k, j))=\frac{x}{(s+1) \zeta(2 r)}+\frac{\log x}{2 \zeta(r)}+L(r ; s)-\frac{1}{s+1} \sum_{d^{r} \leq x} \frac{\mu(d)}{d^{r}} \psi\left(\frac{x}{d^{r}}\right)+\mathcal{O}\left(x^{-1+\frac{1}{r}} \log x\right)
$$

where

$$
L(r ; s)=\frac{1}{2(s+1) \zeta(r)}\left((s+1)\left(\gamma-\frac{r \zeta^{\prime}(r)}{\zeta(r)}\right)-1+2 \sum_{m=1}^{\left\lfloor\frac{s}{2}\right\rfloor}\left(\begin{array}{c}
s+1 \\
2 m
\end{array}\right) B_{2 m} \zeta(2 m)\right) .
$$

Since for all $t \in \mathbb{R}$, it holds that $|\psi(t)| \leq \frac{1}{2}$, we see that the absolute value of the $\psi$-sum is not greater than $\frac{\zeta(r)}{4 \zeta(2 r)}$ and

Also, using Euler's formula

$$
\frac{\zeta(r)}{4 \zeta(2 r)} \leq \frac{5}{4 \pi^{2}}<\frac{2}{5} .
$$

we write

$$
\zeta(2 m)=(-1)^{m+1} 2^{2 m-1} \frac{\pi^{2 m}}{(2 m) !} B_{2 m}
$$

$$
2 \sum_{m=1}^{\left\lfloor\frac{s}{2}\right\rfloor}\left(\begin{array}{c}
s+1 \\
2 m
\end{array}\right) B_{2 m} \zeta(2 m)=\sum_{m=1}^{\left\lfloor\frac{s}{2}\right\rfloor}\left(\begin{array}{c}
s+1 \\
2 m
\end{array}\right)(-1)^{m+1} 2^{2 m} \frac{\pi^{2 m}}{(2 m) !} B_{2 m}^{2}
$$




\section{Acknowledgments}

We thank the anonymous referees for reading this paper carefully and for their valuable suggestions and corrections, which led to a number of improvements in the paper. We also express our gratitude to Olivier Bordellès for his helpful comments on the earlier version of this paper.

\section{References}

[1] D. R. Anderson, T. M. Apostol, The evaluation of Ramanujan's sum and generalizations, Duke Math. J. 20 (1952) $211-216$.

[2] T. M. Apostol, Arithmetical properties of generalized Ramanujan sums, Pacific J. Math. 41 (1972) 281-293.

[3] K. A. Broughan, The average order of the Dirichlet series of the gcd-sum function, J. Integer Seq. 10 (2007) Art\# 07.4.2.

[4] O. Bordellès, The composition of the gcd and certain arithmetic functions, J. Integer Seq. 13 (2010) Art\# 10.7.1.

[5] O. Bordellès, Arithmetic Tales, Springer, Heidelberg, 2012.

[6] K. R. Johnson, An explicit formula for sums of Ramanujan type sums, Indian J. Pure Appl. Math. 18 (1987) 675-677.

[7] I. Kiuchi, Sums of averages of gcd-sum functions, J. Number Theory 176 (2017) 449-472.

[8] I. Kiuchi, On sums of averages of generalized Ramanujan sums, Tokyo J. Math. 40 (2017) 255-275.

[9] I. Kiuchi, M. Minamidem, M. Ueda, Averages of Anderson-Apostol sums, J. Ramanujan Math. Soc. 31 (2016) $339-357$. 